\title{
Blood volume analysis as a guide for dry weight determination in chronic hemodialysis patients: a crossover study
}

\author{
Line Malha', Hasan Fattah², Frank Modersitzki ${ }^{3}$ and David S. Goldfarb ${ }^{4 *}$
}

\begin{abstract}
Background: Volume overload and depletion both lead to high morbidity and mortality. Achieving euvolemia is a challenge in patients with end stage kidney disease on hemodialysis (HD). Blood volume analysis (BVA) uses radiolabeled albumin to determine intravascular blood volume (BV). The measured BV is compared to an ideal BV (validated in healthy controls). We hypothesized that BVA could be used in HD to evaluate the adequacy of the current clinically prescribed "estimated dry weight" (EDW) and to titrate EDW in order to improve overall volume status. We were also interested in the reproducibility of BVA results in end stage kidney disease.

Methods: Twelve adults on chronic HD were recruited; 10 completed the study. BVA (Daxor, New York, NY, USA) was used to measure BV at baseline. EDW was kept the same if the patient was deemed to be euvolemic by BVA otherwise, the prescribed EDW was changed with the aim that measured BV would match ideal BV. A second BVA measurement was done 1-3 months later in order to measure BV again.
\end{abstract}

Results: Based on BVA, 6/10 patients were euvolemic at baseline and 5/10 were euvolemic at the second measurement. When comparing patients who had their prescribed EDW changed after the initial BVA to those who did not, both groups had similar differences between measured and ideal BV $(P=0.75)$. BV values were unchanged at the second measurement $(P=0.34)$ and there was no linear correlation between BV change and weight change $\left(r^{2}=0.08\right)$.

Conclusions: This pilot study is the first longitudinal measurement of BVA in HD patients. It revealed that changing weight did not proportionally change intravascular BV. BV remained stable for 1-3 months. BVA may not be helpful in clinically stable HD patients but studies on patients with hemodynamic instability and uncertain volume status are needed.

Trial registration: ClinicalTrials.gov (NCT02717533), first registered February 4, 2015.

Keywords: Volume control, lodine radioisotopes/diagnostic use, Ultrafiltration, Volume status, Absolute blood volume; dry weight; hemodialysis

\section{Background}

A major purpose of hemodialysis is to remove excess salt and water and restore extracellular fluid volume (ECV) to normal. Solute clearance has been widely used as an objective method to assess dialysis adequacy but there is no equivalent objective assessment for adequate and

\footnotetext{
* Correspondence: david.goldfarb@va.gov

${ }^{4}$ Nephrology Division, NYU School of Medicine and NYU Langone Medical Center, New York University School of Medicine, 423 E. 23 St., New York, NY, USA

Full list of author information is available at the end of the article
}

appropriate volume status. More recently, experts have promoted a "volume first approach". A clear, objective method to assess ECV would be paramount to the success of this approach $[1,2]$.

Chronic volume overload is common in dialysis patients and is often unrecognized by physical exam $[3,4]$ leading clinicians to overestimate dry weight and underestimate the ultrafiltration requirement. Prior studies have demonstrated that in patients with end stage kidney disease (ESKD), volume expansion correlates with elevated blood pressure $[5,6]$ and adjusting the target

(c) The Author(s). 2019 Open Access This article is distributed under the terms of the Creative Commons Attribution 4.0 International License (http://creativecommons.org/licenses/by/4.0/), which permits unrestricted use, distribution, and 
weight leads to improved blood pressure control [7-10]. ECV overload, as determined by bioimpedance [11-14] and lung ultrasound [15], has also been associated with increased mortality and cardiovascular events in chronic kidney disease.

The benefit of additional ultrafiltration and decrease in estimated dry weight (EDW) [7], needs to be weighed against the risks of intradialytic hypotension that can also lead to adverse cardiovascular outcome and mortality [16-18].

There is currently no consensus about which objective measurement of ECV may provide clinically useful and accurate measurement of blood volume (BV) [19] to assist clinicians in finding a safe middle ground between intravascular volume depletion and deleterious ECV expansion. Blood volume analysis (BVA) using radiolabeled albumin is often considered the gold standard to determine intravascular blood volume (BV) and allows comparison to an "ideal" BV [20, 21] (as detailed in the methods section).

BVA has been used with some benefit to evaluate volume status in critically ill [22-25] as well as non-critically ill [26] hospitalized patients. We previously demonstrated that BVA could be used successfully in hemodialysis patients to determine $\mathrm{BV}$, and changes in $\mathrm{BV}$, before and after dialysis, with good correlation with relative BV changes as measured by the online Crit-Line Monitor (CLM III; Hema Metrics, Kaysville, UT, USA) [27].

More recently, Leung et al. have failed to demonstrate a significant difference in hemodialysis outcomes (including intradialytic hypotension) in a randomized clinical trial comparing 32 patient undergoing hemodialysis with and without relative BV monitoring [28]. The lack of benefit from relative BV monitor thus raises the question of the potential benefit of absolute BV measurement, a more accurate indicator of intravascular volume and thus, a more relevant monitoring technique for BV in dialysis patients [29].

In the current study we hypothesized that BVA could be further used in the dialysis unit to evaluate the adequacy of the current clinically prescribed EDW in dialysis patients and to titrate EDW in order to improve overall volume status and decrease the incidence of blood volume misinterpretation. In addition, we were interested in the reproducibility of BVA results in HD patients.

\section{Methods}

\section{Patients}

Eligible patients had ESKD receiving chronic, thrice weekly hemodialysis at the New York Harbor Department of Veterans Affairs Healthcare System (VA-NYHHS). Patients could be enrolled if they were at least 21 years old, able to give informed consent, and willing to have two blood volume measurements performed. We enrolled 12 eligible patients (Fig. 1) between 03/19/14 and 06/03/14. Participants underwent post-hemodialysis BVA as a baseline measurement. Based on their BV status, their prescribed EDW was adjusted (as detailed below). After 1 to 3 months, participants underwent a repeat BV measurement. We compared the second value to the first in order to judge whether an improvement in BV status occurred if the dry weight prescription was changed, or to judge stability if it was not.

Blockade of iodine uptake by the thyroid gland was achieved with a saturated solution of potassium iodide. Participants took $130 \mathrm{mg} /$ day ( 1 drop of $1 \mathrm{~g} / \mathrm{ml}$ potassium iodide solution in $8 \mathrm{oz}$ of water) the day prior to the procedure and for 7 days after the BVA.

The study was approved by the local Institutional Review Board and registered at ClinicalTrials.gov (NCT02717533).

\section{Hemodialysis protocol}

Hemodialysis was performed in accordance with standard, routine clinical care using the Phoenix (Gambro, Stockholm, Sweden) delivery system. The dialyzers used were: Polyflux 210 (Gambro) or Rexeed 25SX (Asahi) as per the patient's prior prescription. Blood flow rates were between 400 and $500 \mathrm{ml} / \mathrm{min}$ and a standard bicarbonate dialysate was used. The treatment time was individualized depending on urea reduction rates and ultrafiltration needs. Vital signs (including blood pressure and heart rate) were recorded at the beginning, during and at the end of the dialysis treatment as per standard practice. Pre- and post- dialysis weights were documented.

Information about intradialytic complications was collected including: intradialytic hypertension (increase in blood pressure $\geq 20 \mathrm{mmHg}$ ), intradialytic hypotension (systolic blood pressure $<90 \mathrm{mmHg}$ or decrease in blood pressure $\geq 20 \mathrm{mmHg}$ ), tachycardia (heart rate $100 / \mathrm{min}$, or change $>30 / \mathrm{min}$ ), cramping and, symptoms of volume depletion (dizziness, weakness, nausea, pre-syncope or syncope) requiring medical intervention (interrupting treatment, adjusting ultrafiltration or administration of normal saline).

\section{Blood volume analysis}

BVA was performed using the BVA-100 device (Daxor Corporation, New York, NY) applying the indicator dilution technique [30]. All BV measurements were made at the VA-NYHHS nuclear medicine area within an hour of ending the participants' hemodialysis session. A blood sample was collected at baseline for evaluation of peripheral hemactocrit (Hct) and background radioactivity. Then, 25 microCuries of Iodine-131 labeled albumin were injected intravenously. After $12 \mathrm{~min}$ (in order to 


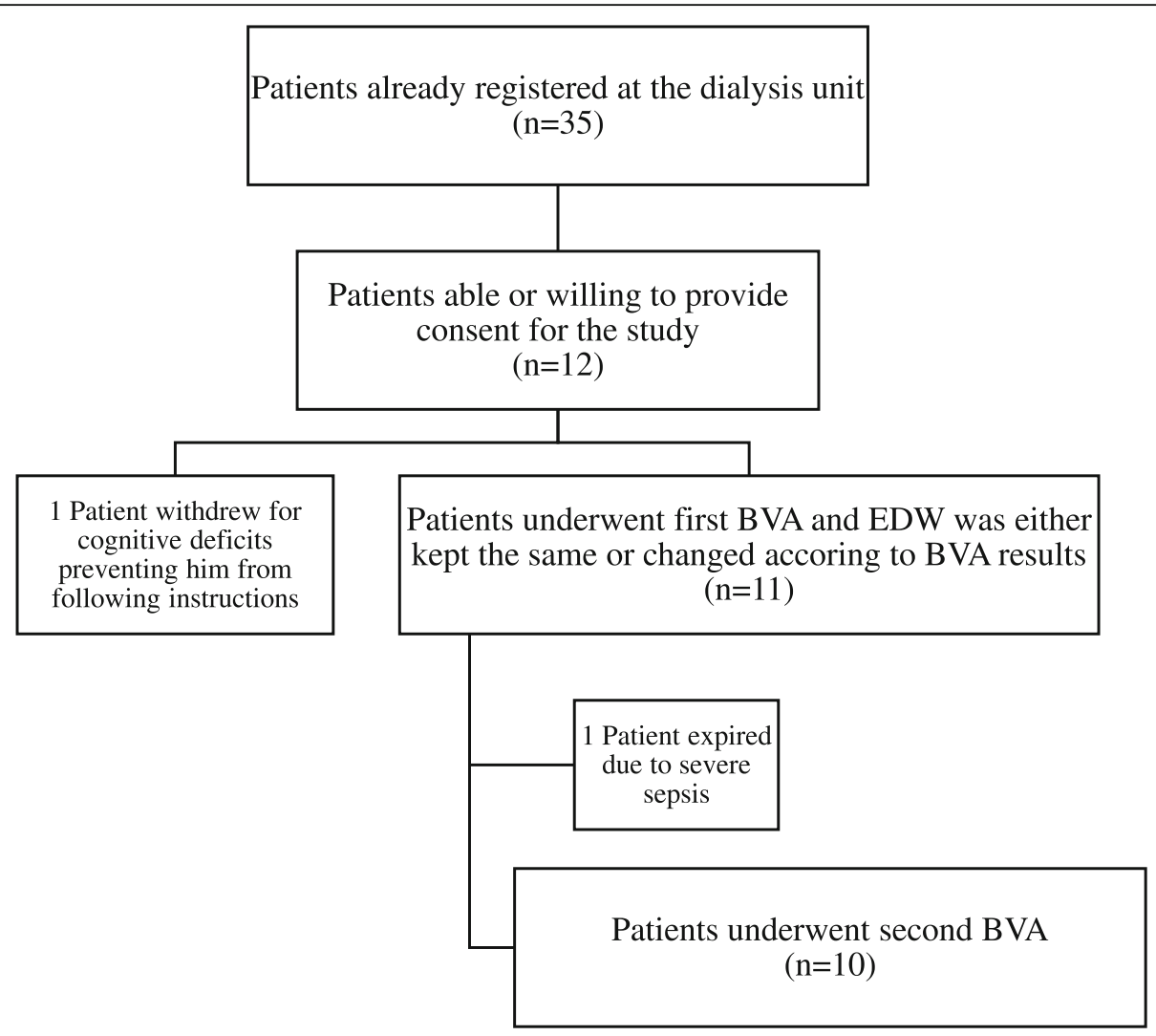

Fig. 1 Participant flow chart. BVA blood volume analysis, EDW estimated dry weight

allow for proper mixing of radiolabeled albumin), $5 \mathrm{ml}$ blood samples were drawn at 6 min intervals for a total of 6 samples. Hct was measured using HemataSTAT $\mathrm{II}^{\circ}$ Microhematocrit System (STI, Sanford, FL) for all samples then; the radioactivity level was measured using the BVA-100 device. Linear regression for radioactivity level of serial samples reflects the transudation of fluid from the intravascular space to the interstitium and allows BV and plasma volume (PV) determination by extrapolation to volume of distribution at time zero.

BVA-100 device software calculates blood volume and plasma volume according to the equations below:

$$
\begin{gathered}
P V=\frac{1000 \times(\text { standard count-baseline count })}{(\text { sample count-baseline count })} \\
B V=\frac{P V}{\left(1-\text { Hct } \times 0.99 \times 0.91 \frac{\text { peripheral Hct }}{\text { whole body Hct }}\right)} .
\end{gathered}
$$

\section{Estimated dry weight adjustment}

Ideal BV is estimated from reference curves derived from the standard Metropolitan Life Insurance Company Desirable Weight tables in order to take into account variability in body habitus [21, 30,31]. For every patient, $\mathrm{BV}$ was measured at baseline and compared to the corresponding ideal BV value. The difference between ideal and measured BV allowed an estimation of the degree of hypervolemia or hypovolemia at baseline, and guided the prescription of EDW. The deviation from ideal BV was considered statistically significant when it is larger than $\pm 8 \%$ as supported by the manufacturer's manual and published literature [21]. The target EDW was defined as the post-dialysis (post-HD) weight at which patient's post-dialysis BV would equal ideal BV. The patient's EDW prescription was changed in order to achieve this target EDW. This definition assumes that ideal BV defines euvolemia for each participant and that $1 \mathrm{~L}$ of BV change would be equivalent to $1 \mathrm{~kg}$ of body weight. Subsequent changes to the prescribed EDW were made according to clinical indications, if needed.

\section{Statistical analysis}

Statistical analysis was performed using Mann-Whitney $\mathrm{U}$ test, Wilcoxon signed rank test and Chi-square test when comparing independent samples. Fisher's exact test was used when comparing 2 groups with less than 5 patients. For paired samples (comparing first to second measurements), Signed rank test was used. Statistical 
testing was computed using the Statistical Package for the Social Sciences (SPSS 20 (SPSS Inc., Chicago). A 2 -tailed $p$-value $<0.05$ was considered to reach statistical significance. Only patients who underwent both BVA measurements were included in the statistical analysis.

\section{Results}

All patients, except for patient number 9, were men with baseline demographics described in Table 1. At baseline, only $3 / 10$ patients were within $0.5 \mathrm{~kg}$ of prescribed EDW. Based on BVA and comparison to "ideal" BV, 6 of 10 patients were euvolemic at baseline (Table 2). Only 1 was judged to be volume overloaded and 3 were considered volume depleted at baseline. Age, blood pressure and heart rate were similar $(p$ value $>0.05)$ for patients that required a change in EDW compared with those who did not.

As per protocol, we then changed the EDW prescribed for the next hemodialysis for these $4 / 10$ patients in order to bring post-HD weight near target EDW (Table 3). We also changed the EDW order for 2 patients who had BV measures suggesting that, although deemed "euvolemic", they were further from ideal BV than might be considered desirable: patient 7 was above ideal BV by $8.0 \%$, and patient 11 was below ideal BV by $-6.2 \%$. These changes were consistent with the protocol and intended to optimize BV. Hemodialysis was then performed with the new target EDW, and BVA was repeated after a variable interval of 1-3 months (Table 1).

Table 1 Patient characteristics

\begin{tabular}{ll}
\hline & Mean \pm Standard Deviation \\
\hline Age & $40 \pm 13$ \\
Years on dialysis & $4.3 \pm 3.2$ \\
Blood pressure (mmHg) & $144 / 78 \pm 28 / 10$ \\
Heart rate (beats/min) & $82 \pm 12$ \\
Deviation in BV (\%) & $-2.7 \pm 9.8$ \\
Time interval between the 2 & $70 \pm 29$ \\
measurements (days) & $\mathrm{N}(\%)$ \\
Gender (Male/Female) & $9 / 1(90 / 10)$ \\
Race & \\
Black & $7(70.0)$ \\
White & $1(10.0)$ \\
$\quad$ Unknown / Not Reported & $2(20.0)$ \\
Ethnicity & \\
Hispanic or Latino & $2(20.0)$ \\
NOT Hispanic or Latino & $8(80.0)$ \\
Was the EDW changed after initial BVA? & \\
No & $4(40.0)$ \\
Yes & $6(60.0)$ \\
\hline
\end{tabular}

The difference between measured BV and ideal BV did not significantly differ from the first to the second BVA measurement ( $P$ value 0.75 ). Patients remained in the same volume status category whether their EDW was changed according to BVA or not ( $\mathrm{P}$ value 0.04). BV remained unchanged at the second measurement (Correlation coefficient $0.81, \mathrm{P}$ value 0.004 ). Among patients whose EDW was kept the same after the BVA measurement, 4/5 were judged to be euvolemic at follow up. The other patient, \#2, had an increase in $\mathrm{BV}$ to $11 \%$ above ideal $\mathrm{BV}$, resulting in a diagnosis of mild volume overload.

The difference between post-HD weight and prescribed EDW was similar between patients who had their EDW changed per protocol and those who did not (P value 0.75 ). There was no linear relationship between the change in absolute post-HD weight and change in $\mathrm{BV}$ in our patient group (Fig. 2).

At baseline, the prevalence of intradialytic complications was low in both groups. No changes in the occurrence of intradialytic hypotension, hypertension or cramps occurred in patients regardless of whether a change in EDW was prescribed. Changing the EDW prescription based on BVA did not affect the occurrence of intradialytic complications. As expected from a prior report [32], there was no apparent association between cramping and iatrogenic volume depletion. Two out of the 3 patients with cramps at a particular session were considered euvolemic for that session while the 3rd patient was deemed volume overloaded.

\section{Discussion}

We previously demonstrated that BVA could be performed in stable HD patients and could yield estimates of BV and correlated with CLM-III readings [27]. Interventional trials have also performed BVA to guide fluid management and diuresis in hospitalized patients $[22,25,26]$.

This study is a pilot to determine feasibility, potential benefits and reproducibility of volume status estimation for outpatients with ESKD on hemodialysis. Despite its feasibility, BVA did not lead to an improvement in achieving euvolemia in our small patient population of chronic HD patients. On the other side, BVA measurements were stable when comparing the first and second measurement; also, patients who did not have a change in EDW per protocol remained euvolemic according to the repeated measurement.

Flythe et al. recently reported an increased mortality for patients who were more than $2 \mathrm{~kg}$ away from prescribed EDW at the end of dialysis sessions [33]; in our study we used a narrower margin and only considered 2 hemodialysis sessions. The difference between EDW and post-HD weight was not affected by changing EDW 


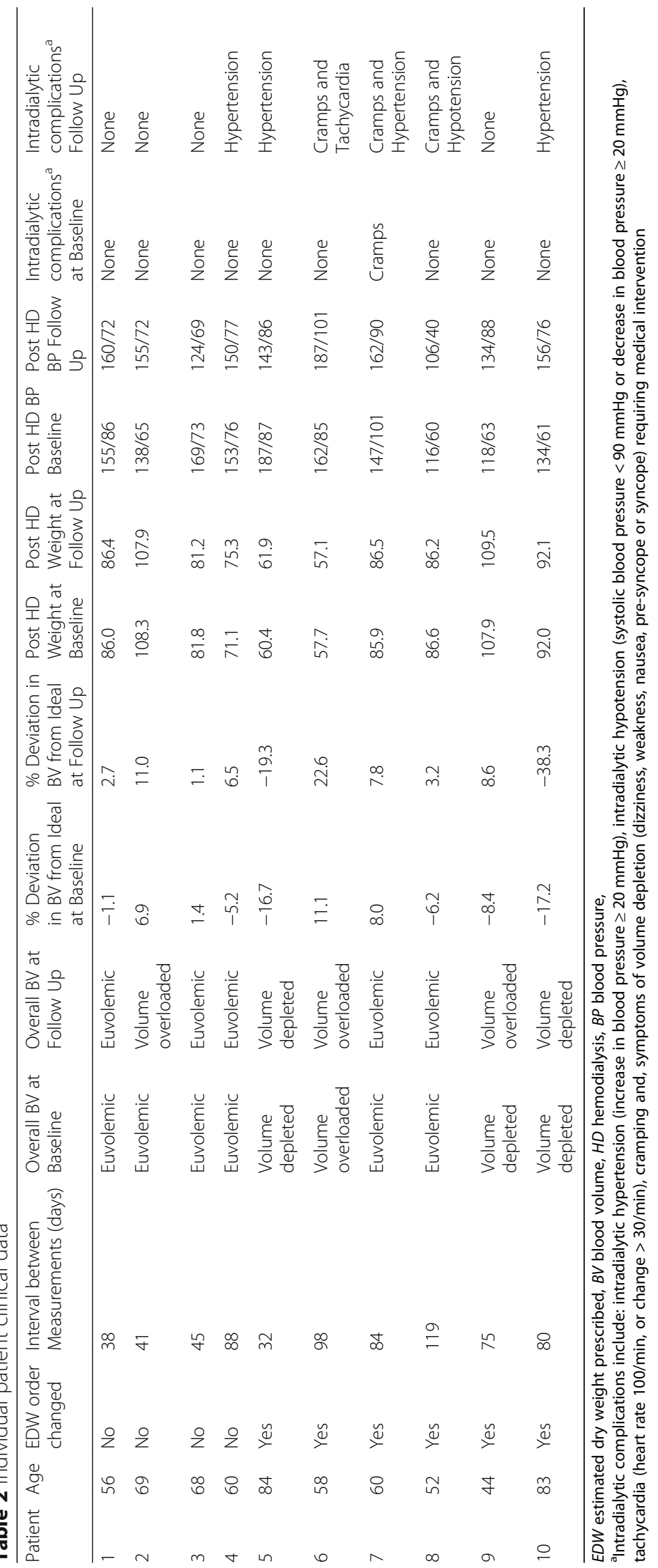




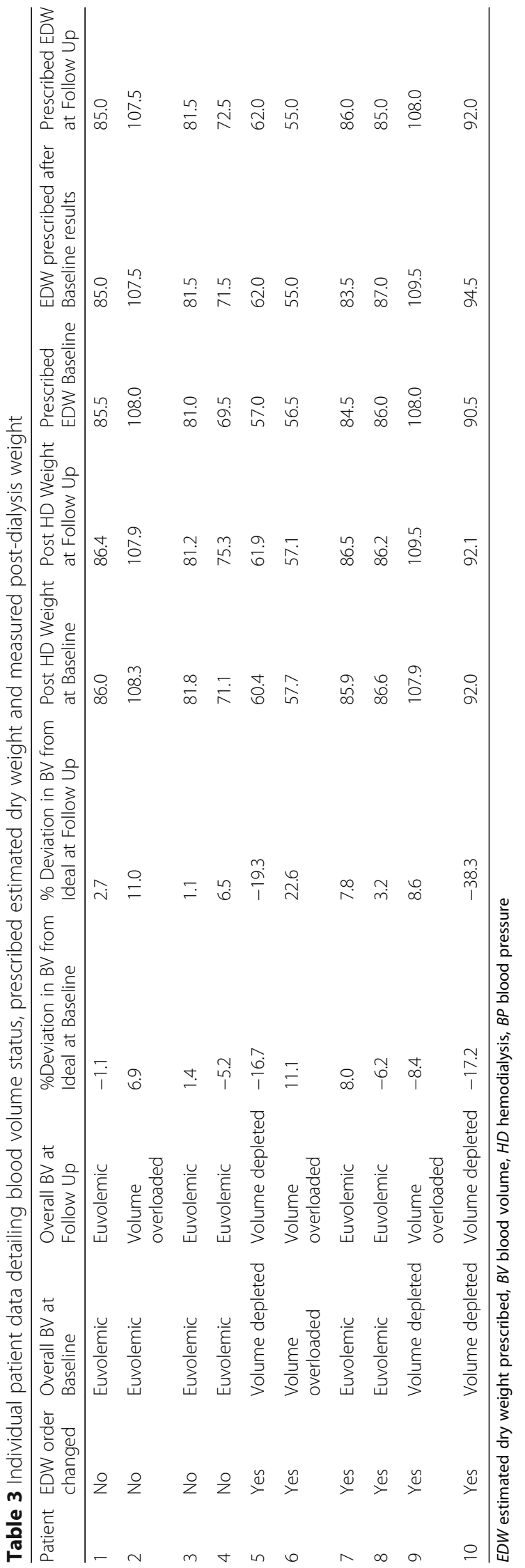




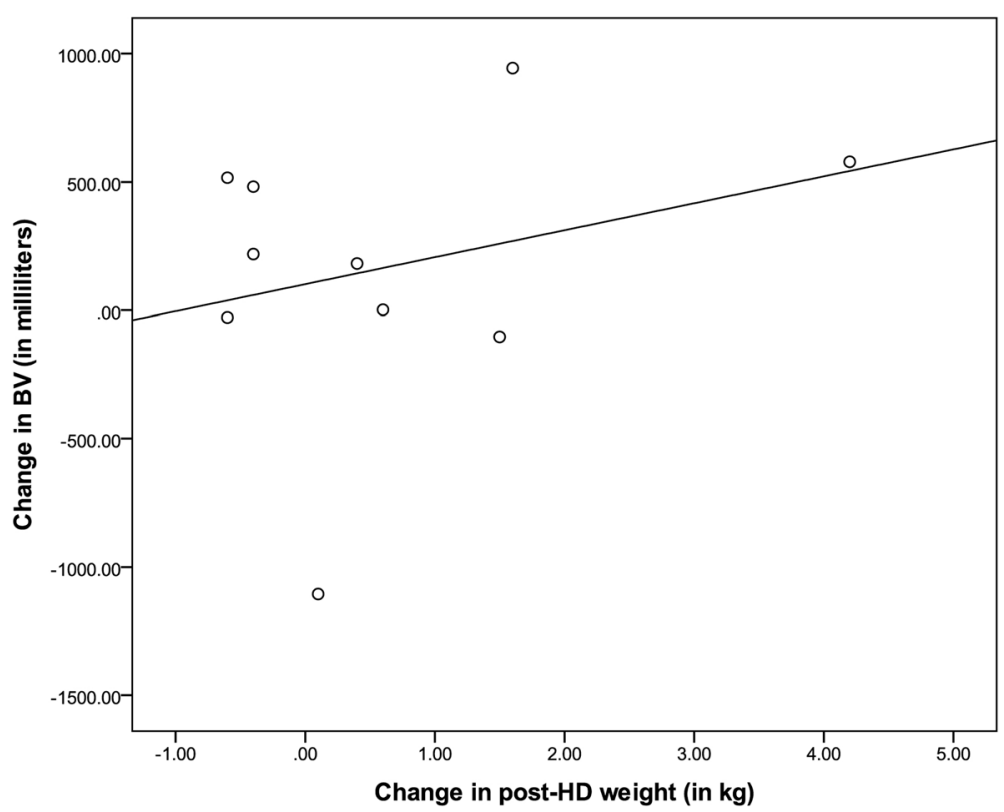

Fig. 2 Change in measured BV between 1st and 2nd BVA (in \%) according to change in measured post dialysis weight (in kg). Change in measured BV(blood volume) (y-axis) is the difference between measured BV at the second BVA (blood volume analysis measurement) and the BV measured at the first BVA. The change in post-HD (post-hemodialysis) (y-axis) weight equals the post-HD weight on the day of the second BVA minus the post-HD weight on the day of the first BVA. Both the change in measured BV and the change in post-HD weights were calculated for every patient. Linear regression was performed and shows no linear relationship between changes in post-HD weight and changes in measured BV (coefficient of determination, $r^{2}=0.08$ )

based on BVA readings and our overall incidence of complication was low as documented in Table $1 .$.

Our protocol was based on the assumption that the observed difference between measured and ideal BV could be extrapolated to a desired change in weight. Based on our data, it is not possible to validate our initial assumption that a change in target weight would affect BV in a linear fashion (Fig. 2). Weight and BV were even found to change in opposite direction in certain cases (Fig. 2). This can be partly explained by the average of 70 days between both measurements, and also by the fact that BVA measures intravascular volume but not total ECV.

Our data are consistent with prior reports showing small changes in BV measurements by BVA in patients with heart failure who were admitted for diuresis despite large changes in body weight upon hospital discharge [26]. Previous BVA data in patients on HD also described a wide variability of the percentage of total weight loss that is in fact from the intravascular component (54 to 99\%) [27]. The heterogeneous source of fluid loss can therefore explain the absence of a linear relationship when several patients are considered in this study.

Our current observation is that despite the variability in post-HD weights, BV measurements were reproducible over 1-3 months in patients on chronic hemodialysis. Thus, a cross-sectional BV measurement may provide information about chronic volume status that is an important factor influencing morbidity in the ESKD population $[6,11,15,34,35]$. This finding further emphasizes the need to realize that a large proportion of fluid removal occurs from interstitial ECV while intravascular ECV appears to be more stable over time and may require a longer-term strategy to be controlled.

Our study has several limitations related to the small sample size and to the fact that participants were generally not far from what was judged to be a desirable or ideal weight. The technique may be more useful for patients newly initiated on HD. BVA may also provide useful information about intravascular ECV to guide management in patients with intradialytic hemodynamic instability.

The second BVA measurement occurred at variable intervals after the first to suit patient and physician preferences. We viewed this as a pragmatic allowance given that the patients were not unstable. The ideal time interval between the initial reading and the follow up measure is currently indeterminate. BVA requires trained personnel, equipment and availability of a nuclear medicine facility (which are possible to obtain in the outpatient setting). The measurement also requires patient cooperation for a time consuming procedure and consent to be subjected to low doses of radiation. Planning 
measurements and repeating BVA should balance patient safety, resource allocation and stability of lean body mass (i.e. non-volume-related body weight).

Non-invasive alternatives to BVA have been developed for determination of volume status in hemodialysis patients. While BVA estimates the whole body's intravascular BV, lung ultrasound evaluates pulmonary congestion and cannot provide information about intravascular fluid or other areas of interstitial fluid accumulation. Inferior vena cava ultrasound measures central vasculature filling and thus only intravascular volume. Bioimpedance spectroscopy (BIS) estimates all extracellular volume and thus cannot appreciate intravascular and interstitial fluid separately. BIS is a promising, non-invasive technique and has been validated as an accurate estimator of extracellular volume against dilution techniques [36, 37]. BIS has been used during HD to with some success to minimize hypotension [38] and improve blood pressure control [8-10].

It is important to keep in mind that BIS and BVA measure different volumes and may be different pieces of the same puzzle to reach "euvolemia". Many patients with ESRD suffer from chronic edema and probing to normalize their total ECV may lead to depletion of intravascular ECV. BVA may be helpful in quantitatively assess the intravascular fluid compartment in these patients and establish an ideal rather than "dry" weight. Many of our patients had a mild increase in BV from ideal, between 1 to $11 \%$ (the normal range is up to $8 \%$ ) and did not have a high incidence of intradialytic complications.

\section{Conclusions}

Our pilot study demonstrates that in a relatively clinically stable population, BV measurements are stable over 1-3 months. The reproducibility of BV measurements despite changes in post-HD weight suggests that most of the fluid removal in dialysis is ultimately pulled from the interstitium rather than intravascular space. BVA may therefore be used to assess chronic volume status rather than acute changes in the ESKD population. Further studies with a more diverse and larger sample population may still be needed in order to further evaluate the relationship between change in weight and change in BV over time. We would look forward to an adequate randomized controlled trial that would be powered to evaluate changes in morbidity after altering EDW according to BVA results and determine an outcome-based target range for BV. Establishing an outcome-based "normal range" for patients of hemodialysis would make BVA a clinically useful test and shift our goal from probing for "dry weight" to identifying clinically safe BV.

\section{Abbreviations}

BIS: Bioimpedance spectroscopy; BV: Blood volume; BVA: Blood volume analysis; ECV: Extracellular fluid volume; EDW: Clinically prescribed "estimated dry weight"; ESKD: End stage kidney disease; Hct: Peripheral hemactocrit; HD: Hemodialysis; post-HD: Post-dialysis; PV: And plasma volume; VANYHHS: New York Harbor Department of Veterans Affairs Healthcare System

\section{Acknowledgements}

None

\section{Funding}

Daxor provided BVA equipment and supplies but was not involved in study design or execution and did not participate in the analysis of data or preparation of the manuscript.

\section{Availability of data and materials}

The data generated and analyzed during the current study are available from the corresponding author on reasonable request.

\section{Disclosures}

Daxor provided BVA equipment and supplies but was not involved in study design or execution and did not participate in the analysis of data or preparation of the manuscript.

\section{Authors' contributions}

DG designed the research protocol. HF and LM collected the patient data. FM and LM analyzed the data. DG and LM have interpreted the data for manuscript production. All the authors have contributed to, reviewed and approved the final manuscript.

\section{Ethics approval and consent to participate}

The study was conducted in accordance with the Declaration of Helsinki and was approved by the local Institutional Review Board, the subcommittee for Human Studies at the VA-NYHHS (protocol number 01249). The patient gave written informed consent upon enrollment in the study.

\section{Consent for publication}

Not applicable.

\section{Competing interests}

Malha, Fattah, Modersitzki: None; Goldfarb: owner, Ravine Group; funding from NIDDK, NCATS. The author declares that there are no competing interests.

\section{Publisher's Note}

Springer Nature remains neutral with regard to jurisdictional claims in published maps and institutional affiliations.

\section{Author details}

${ }^{1}$ Nephrology and Hypertension Division, Weill-Cornell Medicine, 424 East 70th street, New York, NY 10021, USA. ²Nephrology Division, University of Kentucky, UK Transplant Center, 740 S. Limestone, 3rd fl, suite K348, Lexington, KY 40536, USA. ${ }^{3}$ Nephrology Section, New York Harbor VA Healthcare System, Nephrology Section/111G, 423 East. 23 St., New York, NY 10010, USA. ${ }^{4}$ Nephrology Division, NYU School of Medicine and NYU Langone Medical Center, New York University School of Medicine, 423 E. 23 St., New York, NY, USA.

Received: 20 December 2017 Accepted: 14 January 2019

Published online: 11 February 2019

\section{References}

1. Weiner DE, Brunelli SM, Hunt A, Schiller B, Glassock R, Maddux FW, Johnson D, Parker T, Nissenson A. Improving clinical outcomes among hemodialysis patients: a proposal for a "volume first" approach from the chief medical officers of US Dialysis providers. American journal of kidney diseases : the official journal of the National Kidney Foundation. 2014;64(5):685-95.

2. Flythe JE, Brookhart MA: Fluid management: the challenge of defining standards of care. Clin J Am Soc Nephrol : CJASN 2014, 9(12):2033-2035. 
3. Sinha AD, Agarwal R. Can chronic volume overload be recognized and prevented in hemodialysis patients? The pitfalls of the clinical examination in assessing volume status. Semin Dial. 2009;22(5):480-2.

4. Androne AS, Hryniewicz K, Hudaihed A, Mancini D, Lamanca J, Katz SD. Relation of unrecognized hypervolemia in chronic heart failure to clinical status, hemodynamics, and patient outcomes. Am J Cardiol. 2004;93(10): 1254-9.

5. Agarwal R. Volume-associated ambulatory blood pressure patterns in hemodialysis patients. Hypertension. 2009;54(2):241-7.

6. Dionisio P, Valenti M, Bergia R, Caramello E, Stramignoni E, Berto IM, Pellerey M, Bajardi P. Influence of the hydration state on blood pressure values in a group of patients on regular maintenance hemodialysis. Blood Purif. 1997; 15(1):25-33.

7. Agarwal R, Alborzi P, Satyan S, Light RP. Dry-weight reduction in hypertensive hemodialysis patients (DRIP): a randomized, controlled trial. Hypertension. 2009;53(3):500-7.

8. Moissl U, Arias-Guillén M, Wabel P, Fontseré N, Carrera M, Campistol JM, Maduell F. Bioimpedance-guided fluid Management in Hemodialysis Patients. Clin J Am Soc Nephrol. 2013;8(9):1575-82.

9. Machek P, Jirka T, Moissl U, Chamney P, Wabel P. Guided optimization of fluid status in haemodialysis patients. Nephrology, dialysis, transplantation official publication of the European Dialysis and Transplant Association European Renal Association. 2010;25(2):538-44.

10. Onofriescu M, Mardare NG, Segall L, Voroneanu L, Cusai C, Hogas S, Ardeleanu S, Nistor I, Prisada OV, Sascau R, et al. Randomized trial of bioelectrical impedance analysis versus clinical criteria for guiding ultrafiltration in hemodialysis patients: effects on blood pressure, hydration status, and arterial stiffness. Int Urol Nephrol. 2012;44(2):583-91.

11. Wizemann V, Wabel P, Chamney P, Zaluska W, Moissl U, Rode C, MaleckaMasalska T, Marcelli D. The mortality risk of overhydration in haemodialysis patients. Nephrology, dialysis, transplantation : official publication of the European Dialysis and Transplant Association - European Renal Association. 2009;24(5):1574-9.

12. Hung SC, Kuo KL, Peng CH, Wu CH, Lien YC, Wang YC, Tarng DC. Volume overload correlates with cardiovascular risk factors in patients with chronic kidney disease. Kidney Int. 2014;85(3):703-9.

13. Tsai YC, Chiu YW, Tsai JC, Kuo HT, Hung CC, Hwang SJ, Chen TH, Kuo MC, Chen HC: Association of fluid overload with cardiovascular morbidity and all-cause mortality in stages 4 and 5 CKD. Clin J Am Soc Nephrol : CJASN 2015, 10(1):39-46.

14. Chazot C, Wabel P, Chamney P, Moissl U, Wieskotten S, Wizemann V. Importance of normohydration for the long-term survival of haemodialysis patients. Nephrology, dialysis, transplantation : official publication of the European Dialysis and Transplant Association - European Renal Association. 2012;27(6):2404-10.

15. Zoccali C, Tripepi R, Torino C, Bellantoni M, Tripepi G, Mallamaci F. Lung congestion as a risk factor in end-stage renal disease. Blood Purif. 2013; 36(3-4):184-91.

16. Stefansson BV, Brunelli SM, Cabrera C, Rosenbaum D, Anum E, Ramakrishnan $K$, Jensen DE, Stalhammar NO: Intradialytic hypotension and risk of cardiovascular disease. Clin J Am Soc Nephrol : CJASN 2014, 9(12):21242132.

17. Flythe JE, Xue H, Lynch KE, Curhan GC, Brunelli SM. Association of mortality risk with various definitions of intradialytic hypotension. Journal of the American Society of Nephrology : JASN. 2015;26(3):724-34.

18. Daugirdas JT. Measuring intradialytic hypotension to improve quality of care. Journal of the American Society of Nephrology : JASN. 2015;26(3):512-4.

19. Sinha AD. Why assistive technology is needed for probing of dry weight. Blood Purif. 2011:31(1-3):197-202

20. Bhave G, Neilson EG. Body fluid dynamics: back to the future. Journal of the American Society of Nephrology : JASN. 2011;22(12):2166-81.

21. Feldschuh J, Katz $\mathrm{S}$. The importance of correct norms in blood volume measurement. Am J Med Sci. 2007;334(1):41-6.

22. Joffe AM, Khandelwal N, Hallman MR, Treggiari MM. Assessment of circulating blood volume with fluid administration targeting Euvolemia or hypervolemia. Neurocrit Care. 2014.

23. Takahashi EA, Moran SE, Hayashi MS, Inouye DS, Takanishi DM Jr, Yu M. Brain-type natriuretic peptide and right ventricular end-diastolic volume index measurements are imprecise estimates of circulating blood volume in critically ill subjects. The journal of trauma and acute care surgery. 2013; 75(5):813-8
24. Van PY, Riha GM, Cho SD, Underwood SJ, Hamilton GJ, Anderson R, Ham LB, Schreiber MA. Blood volume analysis can distinguish true anemia from hemodilution in critically ill patients. J Trauma. 2011;70(3):646-51.

25. Yu M, Pei K, Moran S, Edwards KD, Domingo S, Steinemann S, Ghows M, Takiguchi S, Tan A, Lurie F, et al. A prospective randomized trial using blood volume analysis in addition to pulmonary artery catheter, compared with pulmonary artery catheter alone, to guide shock resuscitation in critically ill surgical patients. Shock (Augusta Ga). 2011;35(3):220-8.

26. Miller WL, Mullan BP. Understanding the heterogeneity in volume overload and fluid distribution in decompensated heart failure is key to optimal volume management: role for blood volume quantitation. JACC Heart failure. 2014;2(3):298-305.

27. Puri S, Park JK, Modersitzki F, Goldfarb DS. Radioisotope blood volume measurement in hemodialysis patients. Hemodialysis international International Symposium on Home Hemodialysis. 2014;18(2):406-14.

28. Leung KCW, Quinn RR, Ravani P, Duff H, MacRae JM. Randomized crossover trial of blood volume monitoring-guided ultrafiltration biofeedback to reduce intradialytic hypotensive episodes with hemodialysis. Clin J Am Soc Nephrol : CJASN. 2017

29. Hecking M, Schneditz D: Feedback control in hemodialysis-much ado about nothing? Clin J Am Soc Nephrol : CJASN 2017

30. Feldschuh J, Enson Y: Prediction of the normal blood volume. Relation of blood volume to body habitus. Circulation 1977, 56(4 Pt 1):605-612.

31. Manzone TA, Dam HQ, Soltis D, Sagar W. Blood volume analysis: a new technique and new clinical interest reinvigorate a classic study. Journal of nuclear medicine technology. 2007;35(2):55-63 quiz 77, 79.

32. Katz SD. Blood volume assessment in the diagnosis and treatment of chronic heart failure. Am J Med Sci. 2007;334(1):47-52.

33. Flythe JE, Kshirsagar AV, Falk RJ, Brunelli SM. Associations of Posthemodialysis weights above and below target weight with all-cause and cardiovascular mortality. Clin J Am Soc Nephrol. 2015.

34. Agarwal R. Home and ambulatory blood pressure monitoring in chronic kidney disease. Curr Opin Nephrol Hypertens. 2009;18(6):507-12.

35. Enia G, Torino C, Panuccio V, Tripepi R, Postorino M, Aliotta R, Bellantoni M, Tripepi G, Mallamaci F, Zoccali C: Asymptomatic pulmonary congestion and physical functioning in hemodialysis patients. Clin J Am Soc Nephrol: CJASN 2013, 8(8):1343-1348.

36. Raimann JG, Zhu F, Wang J, Thijssen S, Kuhlmann MK, Kotanko P, Levin NW, Kaysen GA. Comparison of fluid volume estimates in chronic hemodialysis patients by bioimpedance, direct isotopic, and dilution methods. Kidney Int. 2014;85(4):898-908.

37. Moissl UM, Wabel P, Chamney PW, Bosaeus I, Levin NW, Bosy-Westphal A, Korth O, Muller MJ, Ellegard L, Malmros V, et al. Body fluid volume determination via body composition spectroscopy in health and disease. Physiol Meas. 2006;27(9):921-33.

38. Maduell F, Arias M, Masso E, Fontsere N, Carrera M, Vera M, Cases A, Campistol JM. Sensitivity of blood volume monitoring for fluid status assessment in hemodialysis patients. Blood Purif. 2013;35(1-3):202-8.

\section{Ready to submit your research? Choose BMC and benefit from:}

- fast, convenient online submission

- thorough peer review by experienced researchers in your field

- rapid publication on acceptance

- support for research data, including large and complex data types

- gold Open Access which fosters wider collaboration and increased citations

- maximum visibility for your research: over $100 \mathrm{M}$ website views per year

At BMC, research is always in progress.

Learn more biomedcentral.com/submissions 